\title{
The role of molecular filaments in the origin of the prestellar core mass function and stellar initial mass function
}

\author{
Ph. André ${ }^{1}$, D. Arzoumanian ${ }^{2,3,1}$, V. Könyves ${ }^{4,1}$, Y. Shimajiri ${ }^{5,1,6}$, and P. Palmeirim ${ }^{3}$ \\ ${ }^{1}$ Laboratoire d'Astrophysique (AIM), CEA/DRF, CNRS, Université Paris-Saclay, Université Paris Diderot, Sorbonne Paris Cité, \\ 91191 Gif-sur-Yvette, France \\ e-mail: pandre@cea.fr \\ 2 Department of Physics, Graduate School of Science, Nagoya University, Furo-cho, Chikusa-ku, Nagoya 464-8602, Japan \\ 3 Instituto de Astrofísica e Ciências do Espaço, Universidade do Porto, CAUP, Rua das Estrelas, 4150-762 Porto, Portugal \\ e-mail: doris .arzoumanian@astro.up.pt \\ 4 Jeremiah Horrocks Institute, University of Central Lancashire, Preston PR1 2HE, UK \\ 5 Department of Physics and Astronomy, Graduate School of Science and Engineering, Kagoshima University, 1-21-35 Korimoto, \\ Kagoshima, Kagoshima 890-0065, Japan \\ ${ }^{6}$ National Astronomical Observatory of Japan, Osawa 2-21-1, Mitaka, Tokyo 181-8588, Japan
}

Received 17 May 2019 / Accepted 29 July 2019

\section{ABSTRACT}

Context. The origin of the stellar initial mass function (IMF) is one of the most debated issues in astrophysics.

Aims. Here we explore the possible link between the quasi-universal filamentary structure of star-forming molecular clouds and the origin of the IMF.

Methods. Based on our recent comprehensive study of filament properties from Herschel Gould Belt survey observations, we derive, for the first time, a good estimate of the filament mass function (FMF) and filament line mass function (FLMF) in nearby molecular clouds. We use the observed FLMF to propose a simple toy model for the origin of the prestellar core mass function (CMF), relying on gravitational fragmentation of thermally supercritical but virialized filaments.

Results. We find that the FMF and the FLMF have very similar shapes and are both consistent with a Salpeter-like power-law function $\left(\mathrm{d} N / \mathrm{d} \log M_{\text {line }} \propto M_{\text {line }}^{-1.5 \pm 0.1}\right)$ in the regime of thermally supercritical filaments $\left(M_{\text {line }}>16 M_{\odot} \mathrm{pc}^{-1}\right)$. This is a remarkable result since, in contrast, the mass distribution of molecular clouds and clumps is known to be significantly shallower than the Salpeter power-law IMF, with $\mathrm{d} N / \mathrm{d} \log M_{\mathrm{cl}} \propto M_{\mathrm{cl}}^{-0.7}$.

Conclusions. Since the vast majority of prestellar cores appear to form in thermally transcritical or supercritical filaments, we suggest that the prestellar CMF and by extension the stellar IMF are at least partly inherited from the FLMF through gravitational fragmentation of individual filaments.

Key words. stars: formation - ISM: clouds - ISM: structure - submillimeter: ISM

\section{Introduction}

The origin of the stellar initial mass function (IMF) is a fundamental problem in modern astrophysics which is still highly debated (e.g., Offner et al. 2014). Two major features of the IMF are a fairly robust power-law slope at the high-mass end (Salpeter 1955) and a broad peak around $\sim 0.3 M_{\odot}$ corresponding to a characteristic stellar mass scale (e.g., Larson 1985). In recent years, the dominant theoretical model proposed to account for these features has been the "gravo-turbulent fragmentation" picture (e.g., Padoan \& Nordlund 2002; Hennebelle \& Chabrier 2008), whereby the properties of supersonic interstellar turbulence lead to the Salpeter power law, while gravity and thermal physics set the characteristic mass scale (see Larson 2005). This picture is deterministic in the sense that stellar masses are directly inherited from the distribution of prestellar core masses resulting from cloud fragmentation prior to protostellar collapse, in agreement with the observed similarity between the prestellar core mass function (CMF) and the system IMF (e.g., Motte et al. 1998; Alves et al. 2007; Könyves et al. 2015).
In contrast, a major alternative view posits that stellar masses are essentially unrelated to initial prestellar core masses and result entirely from stochastic competitive accretion and dynamical interactions between protocluster seeds at the protostellar stage (Class 0/Class I) of young stellar object evolution (Bonnell et al. 2001; Bate et al. 2003). Here, we discuss modifications to the gravo-turbulent picture based on Herschel results in nearby molecular clouds, which emphasize the importance of filaments in the star formation process and potentially the CMF and IMF (e.g., André et al. 2010).

Herschel imaging observations have shown that filamentary structures are truly ubiquitous in the cold interstellar medium (ISM) of the Milky Way (Molinari et al. 2010), dominate the mass budget of Galactic molecular clouds at high densities $\left(\gtrsim 10^{4} \mathrm{~cm}^{-3}\right.$ ) (Schisano et al. 2014; Könyves et al. 2015), and feature a high degree of universality in their properties. In particular, detailed analysis of the radial column density profiles indicates that, at least in the nearby clouds of the Gould Belt, molecular filaments are characterized by a narrow distribution of crest-averaged inner widths with a typical full width at 

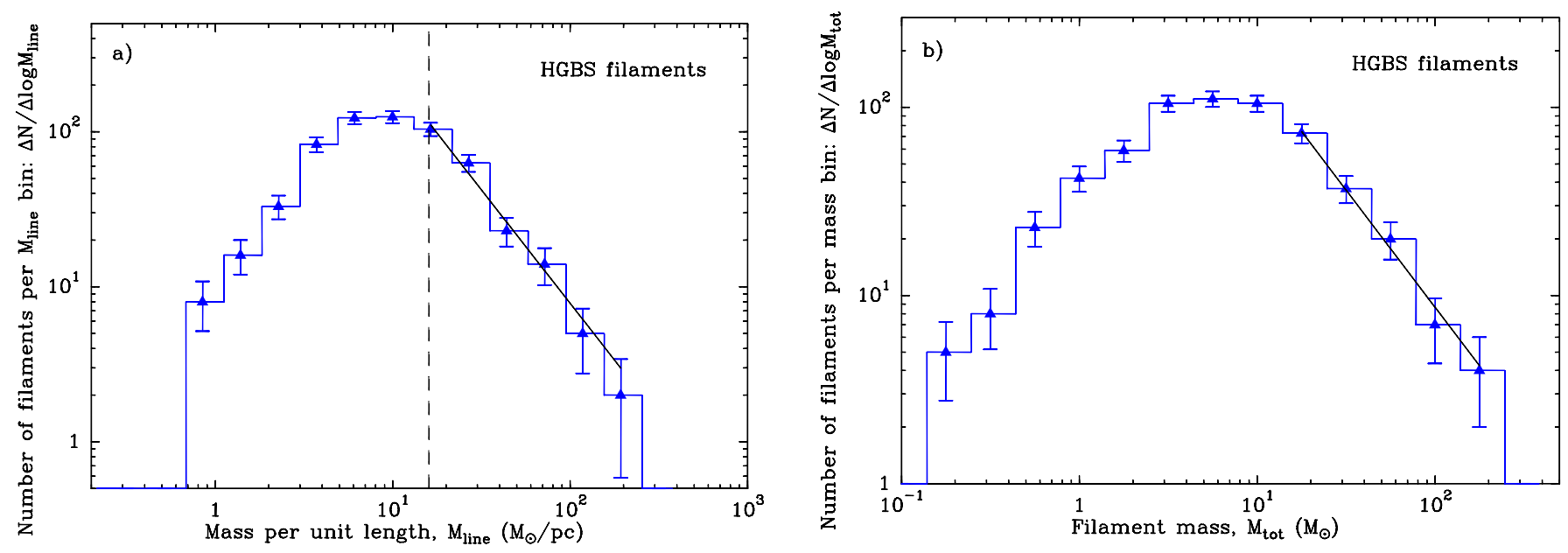

Fig. 1. Panel a: differential distribution of crest-averaged masses per unit length for the sample of 599 robust filaments identified by Arzoumanian et al. (2019) in the Herschel GBS maps of eight nearby molecular clouds (IC5146, Orion B, Aquila, Musca, Polaris, Pipe, Taurus L1495, and Ophiuchus). Above the critical mass per unit length $M_{\text {line,crit }} \sim 16 M_{\odot} \mathrm{pc}^{-1}$ (vertical dashed line), the filament sample is estimated to be more than $90 \%$ complete (see text) and the distribution is well fitted by a Salpeter-like power law $\Delta N / \Delta \log M_{\text {line }} \propto M_{\text {line }}^{-1.6 \pm 0.1}$ (solid line segment). Panel $b$ : differential distribution of total masses for the same sample of filaments as in the left panel. At the high-mass end $\left(M_{\text {tot }}>15 M_{\odot}\right)$, the distribution of filament masses is well fitted by a Salpeter-like power law $\Delta N / \Delta \log M_{\mathrm{tot}} \propto M_{\mathrm{tot}}^{-1.4 \pm 0.1}$ (solid line segment). In both panels, the error bars correspond to $\sqrt{N}$ counting uncertainties.

half maximum (FWHM) value $W_{\text {fil }} \sim 0.1 \mathrm{pc}$ and a dispersion of less than a factor of $\sim 2$ (Arzoumanian et al. 2011, 2019; Koch \& Rosolowsky 2015). Another major result from Herschel (e.g., André et al. 2010; Könyves et al. 2015; Marsh et al. 2016) is that the vast majority $(>75 \%)$ of prestellar cores are found in dense "transcritical" or "supercritical" filaments for which the mass per unit length, $M_{\text {line }}$, is close to or exceeds the critical line mass of nearly isothermal, long cylinders (e.g., Inutsuka \& Miyama 1997), $M_{\text {line,crit }}=2 c_{\mathrm{s}}^{2} / G \sim 16 M_{\odot} \mathrm{pc}^{-1}$, where $c_{\mathrm{s}} \sim 0.2 \mathrm{~km} \mathrm{~s}^{-1}$ is the isothermal sound speed for molecular gas at $T \sim 10 \mathrm{~K}$. Moreover, most prestellar cores lie very close to the crests (i.e., within the inner $0.1 \mathrm{pc}$ portion) of their parent filaments (e.g., Könyves et al. 2019; Ladjelate et al. 2019). These findings support a filamentary paradigm in which lowmass star formation occurs in two main steps (André et al. 2014; Inutsuka et al. 2015): (1) multiple large-scale compressions of cold interstellar material in supersonic magneto-hydrodynamic (MHD) flows generate a cobweb of $\sim 0.1 \mathrm{pc}$-wide filaments within sheet-like or shell-like molecular gas layers in the ISM and (2) the densest molecular filaments fragment into prestellar cores (and then protostars) by gravitational instability near or above the critical line mass, $M_{\text {line,crit }}$, corresponding to $\Sigma_{\text {gas }}^{\text {crit }} \sim$ $M_{\text {line,crit }} / W_{\text {fil }} \sim 160 M_{\odot} \mathrm{pc}^{-2}$ in gas surface density $\left(A_{V} \sim 7.5\right)$ or $n_{\mathrm{H}_{2}} \sim 2 \times 10^{4} \mathrm{~cm}^{-3}$ in volume density. This paradigm differs from the classical gravo-turbulent picture (Mac Low \& Klessen 2004 ) in that it relies on the anisotropic formation of dense structures (such as shells, filaments, cores) in the cold ISM and the unique properties of filamentary geometry (see Larson 2005).

In the present paper, we exploit the results of our recent comprehensive study of filament properties from Herschel Gould Belt survey (HGBS) observations (Arzoumanian et al. 2019) and argue that the distribution of filament masses per unit length may directly connect to the CMF and by extension the IMF. Section 2 presents our observational results on the filament line mass function. Section 3 discusses potential implications of these results for the origin of the prestellar CMF. Section 4 discusses the possible origin of the filament line mass function and concludes the paper.

\section{Observations of the filament line mass function}

Arzoumanian et al. (2019) recently presented a census of filament structures observed with Herschel in eight nearby regions covered by the HGBS: IC5146, Orion B, Aquila, Musca, Polaris, Pipe, Taurus L1495, and Ophiuchus. Using the DisPerSE algorithm (Sousbie 2011) to trace filaments in the HGBS column density maps of these eight clouds ${ }^{1}$, they identified a total of 1310 filamentary structures, including a selected sample of 599 robust filaments with aspect ratio (length/width) $>3$ and central column density contrast $\delta \Sigma_{\text {fil }} / \Sigma_{\text {cloud }}>30 \%$ (where $\delta \Sigma_{\text {fil }}$ is the background-subtracted gas surface density of the filament and $\Sigma_{\text {cloud }}$ the surface density of the parent cloud). Performing an extensive set of tests on synthetic data, Arzoumanian et al. (2019, see their Appendix A) estimated their selected sample of 599 filaments to be more than $95 \%$ complete (and contaminated by less than $5 \%$ of spurious detections) for filaments with column density contrast $\geq 100 \%$. For reference, the column density contrast of isothermal model filaments in pressure equilibrium with their parent cloud is $\left\langle\delta \Sigma_{\text {fil }}\right\rangle / \Sigma_{\text {cloud }} \approx 1.18 \times \sqrt{f_{\text {cyl }} /\left(1-f_{\text {cyl }}\right)}$, where $f_{\text {cyl }} \equiv M_{\text {line }} / M_{\text {line,crit }}<1$ (see Fischera \& Martin 2012) ${ }^{2}$. Thermally transcritical filaments with $M_{\text {line,crit }} / 2 \lesssim M_{\text {line }}<$ $M_{\text {line,crit }}$ (i.e., $f_{\text {cyl }} \gtrsim 0.5$ ) are therefore expected to have column density contrasts $\gtrsim 100 \%$, while thermally supercritical filaments with well-developed power-law density profiles reach column density contrasts $\gg 100 \%$. The selected sample of Arzoumanian et al. (2019) is thus estimated to be $>95 \%$ complete to thermally supercritical filaments with $M_{\text {line }}>M_{\text {line,crit }} \sim$ $16 M_{\odot} \mathrm{pc}^{-1}$.

The differential distribution of average masses per unit length, or filament line mass function (FLMF), derived from Herschel data for the 599 filaments of this sample is shown in Fig. 1a. It can be seen that the FLMF is consistent with a power-law distribution in the supercritical mass per unit length

1 The corresponding column density maps and derived fila-
ment skeleton maps are available in fits format from http://
gouldbelt-herschel. cea.fr/archives

2 Equilibrium model filaments exist only for subcritical masses per unit length, i.e., $f_{\text {cyl }} \leq 1$. 

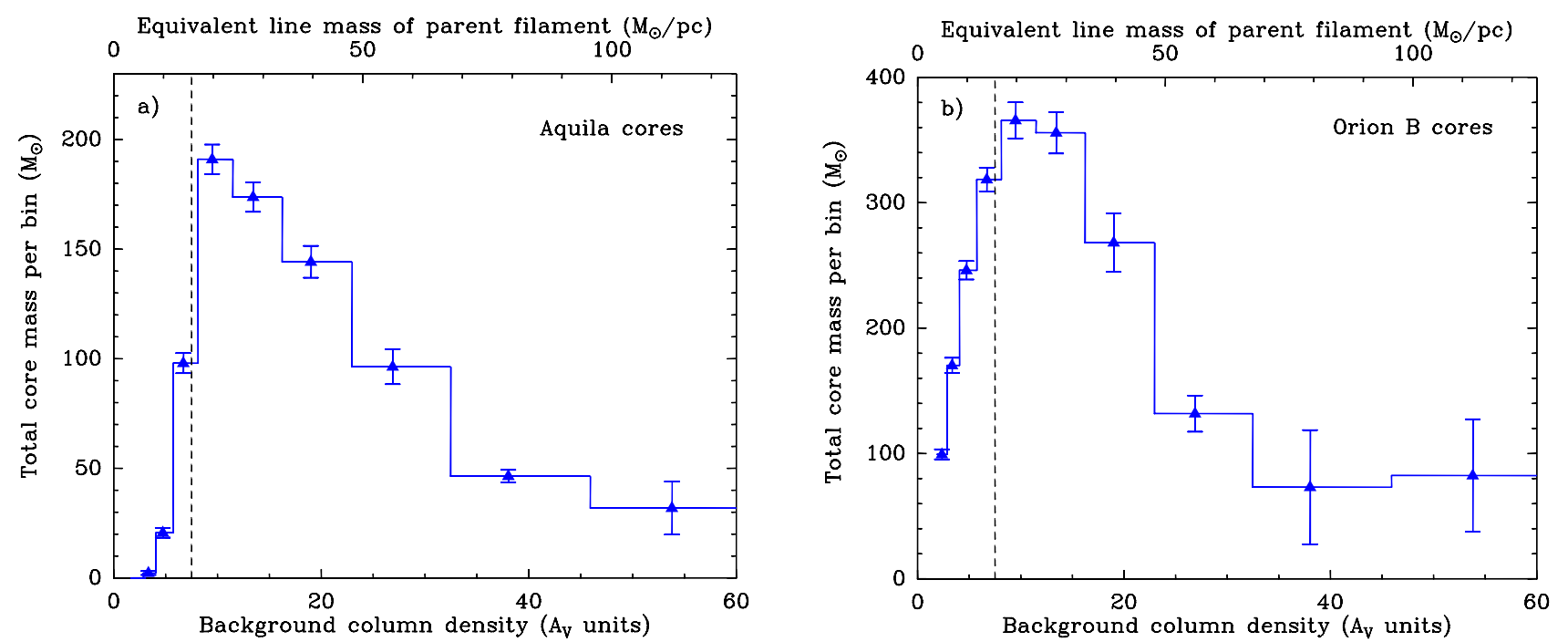

Fig. 2. Total mass in the form of prestellar cores as a function of background column density (lower $x$-axis, in units of $10^{21} \mathrm{H}_{2} \mathrm{~cm}^{-2}$ ) or equivalent mass per unit length of the parent filament (upper $x$-axis, in units of $M_{\odot} \mathrm{pc}^{-1}$ ) for the Aquila cloud (left panel; based on Könyves et al. 2015) and Orion B cloud (right panel; adapted from Könyves et al. 2019). The vertical dashed line marks the fiducial threshold for the formation of prestellar cores at a background $A_{V}=7.5$, equivalent to a mass per unit length of $\sim 16 M_{\odot} \mathrm{pc}^{-1}$, assuming parent filaments of $0.1 \mathrm{pc}$ in width.

regime (above $16 M_{\odot} \mathrm{pc}^{-1}$ ), $\Delta N / \Delta \log M_{\text {line }} \propto M_{\text {line }}^{-1.59 \pm 0.07}$, at a Kolmogorov-Smirnov (K-S) significance level of $92 \%$. The error bar on the power-law exponent was derived by performing a non-parametric K-S test (see, e.g., Press et al. 1992) on the cumulative distribution of masses per unit length $N\left(>M_{\text {line }}\right)$, and corresponds to the range of exponents for which the K-S significance level is larger than $68 \%$ (equivalent to $1 \sigma$ in Gaussian statistics). Remarkably, the FLMF function observed above $M_{\text {line,crit }} \sim 16 M_{\odot} \mathrm{pc}^{-1}$ is very similar to the Salpeter power-law IMF (Salpeter 1955), which scales as $\mathrm{d} N / \mathrm{d} \log M_{\star} \propto M_{\star}^{-1.35}$ in the same format.

The right panel of Fig. 1 shows the distribution of total masses, integrated over filament length, for the same sample of filaments. As can be seen in Fig. 1a, this filament mass function (FMF) is very similar in shape to the FLMF of Fig. 1a, and is also consistent with Salpeter-like power-law distribution at the high-mass end $\left(M_{\text {tot }}>15 M_{\odot}\right), \Delta N / \Delta \log M_{\text {tot }} \propto M_{\text {tot }}^{-1.38 \pm 0.10}$, at a Kolmogorov-Smirnov (K-S) significance level of $98 \%$. The similarity between the FMF and the FLMF is not surprising since $M_{\text {tot }}=M_{\text {line }} \times L$ and the lengths $L$ of the filaments in the Arzoumanian et al. (2019) sample have an approximately lognormal distribution centered at approximately $0.5-$ $0.6 \mathrm{pc}$ (see Fig. A.1a), with no correlation with $M_{\text {line }}$ (the linear Pearson correlation coefficient between $L$ and $M_{\text {line }}$ is $|\rho|<8 \%$ ). Accordingly, a strong linear correlation exists between $M_{\text {tot }}$ and $M_{\text {line }}$ in the filament sample (correlation coefficient $>75 \%$; see Fig. A.1b). We note, however, that the estimated FMF shown in Fig. 1b should be interpreted with caution and is not as robust as the FLMF in Fig. 1a because filament-finding algorithms, such as DisPerSE used in the present analysis or getfilaments (Men'shchikov 2013), tend to break up filamentary structures into small filament segments.

\section{Role of filaments in the prestellar CMF}

At least in terms of mass, most prestellar cores appear to form just above the fiducial column density "threshold" at $A_{V} \sim 7.5$, corresponding to marginally thermally supercritical filaments with $M_{\text {line }} \gtrsim 16 M_{\odot} \mathrm{pc}^{-1}$ (Könyves et al. 2019; see also Fig. 2). In the observationally driven filamentary paradigm of star formation supported by Herschel results (see Sect. 1), the dense cores making up the peak of the prestellar CMF, presumably related to the peak of the IMF, originate from gravitational fragmentation of filaments near the critical threshold for cylindrical gravitational instability (André et al. 2014). In this picture, the characteristic prestellar core mass roughly corresponds to the local Jeans mass in transcritical or marginally supercritical filaments. The thermal Jeans or critical Bonnor-Ebert mass (e.g., Bonnor 1956) is $M_{\mathrm{BE}, \mathrm{th}} \approx 1.18 c_{\mathrm{s}}^{4} /\left(G^{3 / 2} P_{\mathrm{cl}}^{1 / 2}\right)$, where $P_{\mathrm{cl}}$ is the local pressure of the ambient cloud. The latter may be expressed as a function of cloud column density, $\Sigma_{\mathrm{cl}}$, as $P_{\mathrm{cl}} \approx 0.88 G \Sigma_{\mathrm{cl}}^{2}$ (McKee \& Tan 2003). Within a critical $\sim 0.1$ pc-wide filament at $\sim 10 \mathrm{~K}$ with $M_{\text {line }} \approx M_{\text {line,crit }} \sim 16 M_{\odot} \mathrm{pc}^{-1}$ and surface density $\Sigma_{\text {fil }} \approx \Sigma_{\text {gas }}^{\text {crit }} \sim 160 M_{\odot} \mathrm{pc}^{-2}$ (see Sect. 1), the local Bonnor-Ebert mass is thus

$M_{\mathrm{BE}, \mathrm{th}} \sim 1.3 \frac{c_{\mathrm{s}}^{4}}{G^{2} \Sigma_{\mathrm{fil}}} \sim 0.5 M_{\odot} \times\left(\frac{T}{10 \mathrm{~K}}\right)^{2} \times\left(\frac{\Sigma_{\mathrm{fil}}}{160 M_{\odot} \mathrm{pc}^{-2}}\right)^{-1}$

This corresponds very well to the peak of the prestellar CMF at $\sim 0.6 M_{\odot}$ observed in the Aquila cloud (Könyves et al. 2015) and is also consistent within a factor of $<2$ with the CMF peak found with Herschel in other nearby regions such as Taurus L1495 (Marsh et al. 2016) or Ophiuchus (Ladjelate et al. 2019).

The fragmentation of purely thermal equilibrium filaments may be expected to result in a narrow ( $\delta$-like) prestellar CMF sharply peaked at the median thermal Jeans mass (see Lee et al. 2017). However, at least two effects contribute to broadening the observed CMF. First, the filament formation process through multiple large-scale compressions generates a field of initial density fluctuations within star-forming filaments (Inutsuka 2001; Inutsuka et al. 2015). Based on a study of the density fluctuations observed with Herschel along a sample of 80 subcritical or marginally supercritical filaments in three nearby clouds, Roy et al. (2015) found that the power spectrum of line-mass fluctuations is well fitted by a power law, $P(k) \propto k^{\alpha}$ with $\alpha=$ $-1.6 \pm 0.3$. This is consistent with the $1 \mathrm{D}$ power spectrum generated by subsonic Kolmogorov turbulence $(\alpha=-5 / 3)$. Starting from such an initial power spectrum, the theoretical analysis by Inutsuka (2001) shows that the density perturbations quickly 


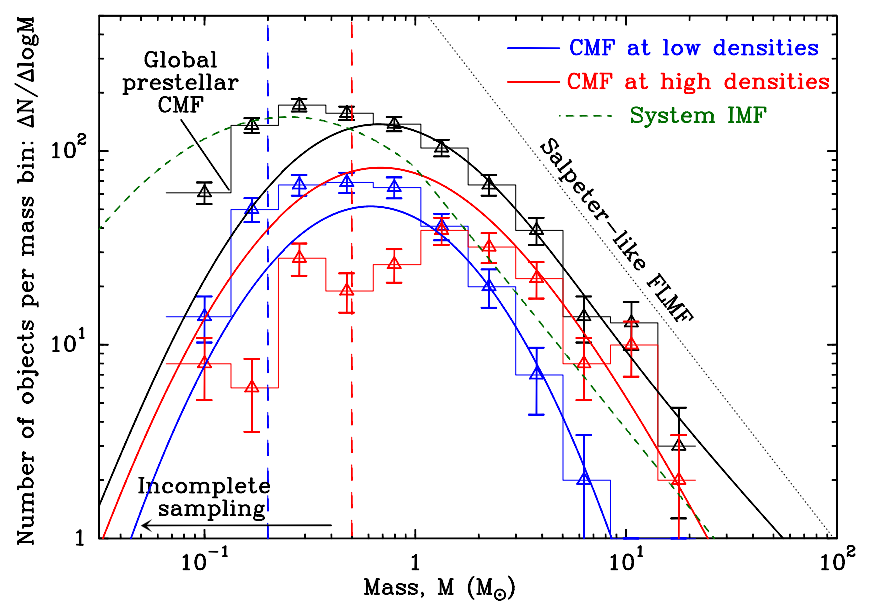

Fig. 3. Comparison of the prestellar CMFs (in $\Delta N / \Delta \log M$ format) expected in the toy model described in the text (solid curves) with the prestellar CMFs observed in the Orion B cloud complex (data points and histograms, adapted from Könyves et al. 2019) at low column densities $\left(4<A_{V}^{\text {back }}<7.5\right.$, in blue $)$, higher column densities $\left(7.5<A_{V}^{\text {back }}<21\right.$, in red), and overall (all $A_{V}^{\text {back }}$, in black). The black dotted line displays the Salpeter-like power law FLMF, $\mathrm{d} N / \mathrm{d} \log M_{\text {line }} \propto M_{\text {line }}^{-1.4}$, assumed in the toy model and consistent with the observed FLMF in the supercritical regime (see Fig. 1). The green dashed curve shows the system IMF (Chabrier 2005, see also Kroupa 2001). The two vertical dashed lines mark the estimated $80 \%$ completeness limits of the Herschel census of prestellar cores in Orion B at low and high background column densities respectively (see Könyves et al. 2019). The CMF extends to higher masses at higher column densities, i.e., higher $M_{\text {line }}$ filaments in both the toy model and the observations.

evolve (in about two free-fall times or $\sim 0.5 \mathrm{Myr}$ for a critical $0.1 \mathrm{pc}$-wide filament) from a mass distribution similar to that of CO clumps (Kramer et al. 1998) to a population of protostellar cores whose mass distribution approaches the Salpeter power law at the high-mass end. However, this process alone is unlikely to produce a CMF with a well-developed Salpeter-like powerlaw tail since very long filaments would be required.

A second broadening effect is due to the power-law distribution of filament masses per unit length (FLMF) in the supercritical regime (Fig. 1a). Given the typical filament width $W_{\text {fil }} \sim 0.1 \mathrm{pc}$ (Arzoumanian et al. 2011, 2019) and the fact that thermally supercritical filaments are observed to be approximately virialized with $M_{\text {line }} \sim \Sigma_{\text {fil }} \times W_{\text {fil }} \sim M_{\text {line,vir }} \equiv 2 c_{\text {s,eff }}^{2} / G$, where $c_{\mathrm{s}, \text { eff }}$ is the one-dimensional velocity dispersion or effective sound speed (Fiege \& Pudritz 2000; Arzoumanian et al.2013) ${ }^{3}$, the effective Bonnor-Ebert mass $M_{\mathrm{BE}, \text { eff }} \sim 1.3 c_{\mathrm{s} \text {,eff }}^{4} /\left(G^{2} \Sigma_{\mathrm{fil}}\right)$ scales roughly as $\Sigma_{\text {fil }}$ or $M_{\text {line. }}$. At the same time, the thermal Bonnor-Ebert mass $M_{\mathrm{BE}, \mathrm{th}}$ scales roughly as $\Sigma_{\text {fil }}^{-1}$ or $M_{\text {line }}^{-1}$ (see Eq. (1)). Hence, both higher- and lower-mass cores may form in higher $M_{\text {line }}$ filaments. In agreement with this expected trend, dense cores of median mass $\sim 10 M_{\odot}$, i.e., an order of magnitude higher that the peak of the prestellar CMF in low-mass nearby filaments (see above and Fig. 3), have recently been detected with ALMA in the NGC 6334 main filament, which is an order of magnitude denser and more massive $\left(M_{\text {line }} \sim 1000 M_{\odot} \mathrm{pc}^{-1}\right)$ than the Taurus B211/B213 filament and other Gould Belt filaments (Shimajiri et al. 2019a). Furthermore, observations indicate that the prestellar CMF tends

\footnotetext{
3 Assuming rough equipartition between magnetic energy and kinetic energy, thermally supercritical filaments may also be close to magnetohydrostatic equilibrium since the magnetic critical line mass $M_{\text {line,crit }}^{\mathrm{mag}}$ may largely exceed $M_{\text {line,crit }}$ (see Tomisaka 2014).
}

to be broader at higher ambient cloud column densities, i.e., in denser parent filaments (Könyves et al. 2019; see also Fig. 3). Since the characteristic fragmentation mass $M_{\mathrm{BE} \text {,eff }}$ scales linearly with $M_{\text {line }}$, we may expect the Salpeter-like distribution of line masses observed above $M_{\text {line,crit }}$ (Fig. 1a) to directly translate into a Salpeter-like power-law distribution of characteristic core masses. In detail, the global prestellar CMF results from the convolution of the CMF produced by individual filaments with the FLMF (see Lee et al. 2017).

Based on the Herschel results and these qualitative considerations, we propose the following observationally driven quantitative scenario to illustrate the potential key role of the FLMF in the origin of the global prestellar CMF in molecular clouds. We assume that all prestellar cores form in thermally transcritical or supercritical (but virialized) filaments and that the outcome of filament fragmentation depends only on the line mass of the parent filament. We denote by $f_{M_{\text {line }}}(m) \equiv \mathrm{d} N_{M_{\text {line }}} / \mathrm{d} \log m$ the differential CMF (per unit log mass, where $m$ represents core mass) in a filament of line mass $M_{\text {line }}$. While the exact form of $f_{M_{\text {line }}}(m)$ is observationally quite uncertain, the foregoing arguments suggest that it should present a peak around the effective Bonnor-Ebert mass $M_{\mathrm{BE} \text {,eff }}$ and may have a characteristic width scaling roughly as the ratio $M_{\mathrm{BE}, \mathrm{eff}} / M_{\mathrm{BE}, \mathrm{th}}$. We thus make the minimal assumption that $f_{M_{\text {line }}}(m)$ follows a lognormal distribution centered at $M_{\mathrm{BE}, \text { eff }}\left(M_{\text {line }}\right)$ and of standard deviation $\sigma_{M_{\text {line }}}\left(M_{\mathrm{BE}, \text { eff }} / M_{\mathrm{BE}, \mathrm{th}}\right)$ in $\log m$ :

$f_{M_{\text {line }}}(m)=A \times \exp \left(-\frac{\left(\log m-\log M_{\mathrm{BE}, \mathrm{eff}}\right)^{2}}{2 \sigma_{M_{\text {line }}}^{2}}\right)$.

We tested various simple functional forms for $\sigma_{M_{\text {line }}}\left(M_{\mathrm{BE}, \text { eff }} / M_{\mathrm{BE}, \text { th }}\right)$ and adopted $\sigma_{M_{\text {line }}}^{2}=0.4^{2}+0.3\left[\log \left(M_{\mathrm{BE}, \text { eff }} /\right.\right.$ $\left.\left.M_{\mathrm{BE}, \mathrm{th}}\right)\right]^{2}$ as an illustrative fiducial form providing a reasonable good match to the observational constraints (see Fig. 3 and Appendix B).

Denoting by $g\left(M_{\text {line }}\right) \equiv \mathrm{d} N / \mathrm{d} \log M_{\text {line }}$ the differential FLMF per unit log line mass, the global prestellar CMF per unit log mass $\xi(m) \equiv \mathrm{d} N_{\text {tot }} / \mathrm{d} \log m$ may be obtained as a weighted integration over line mass of the CMFs in individual filaments:

$\xi(m)=\int f_{M_{\text {line }}}(m) \times w\left(M_{\text {line }}\right) \times g\left(M_{\text {line }}\right) \times \operatorname{dlog} M_{\text {line }}$,

where $w\left(M_{\text {line }}\right) \propto \operatorname{CFE}\left(M_{\text {line }}\right) \times M_{\text {line }} \times L$ represents the relative weight as a function of $M_{\text {line }}, \operatorname{CFE}\left(M_{\text {line }}\right)$ is the prestellar core formation efficiency, and $L$ the filament length. The results given in Sect. 2 suggest that the FLMF is a power law $g\left(M_{\text {line }}\right) \propto M_{\text {line }}^{-\alpha}$ with $\alpha \approx 1.4$. As $L$ and $M_{\text {line }}$ are not correlated in the filament sample of Arzoumanian et al. (2019) (Sect. 2), here we adopt $L=$ constant $\sim 0.55$ pc for simplicity (see Fig. A.1). Observationally, $\operatorname{CFE}\left(M_{\text {line }}\right)$ exhibits a sharp transition between a regime of negligible prestellar core formation efficiency at $M_{\text {line }} \ll M_{\text {line,crit }}$ and a regime of roughly constant core formation efficiency $\sim 15-$ $20 \%$ at $M_{\text {line }} \gg M_{\text {line,crit }}$ (see Sect. 1). Following Könyves et al. (2015), we describe this transition as a smooth step function of the form $\operatorname{CFE}\left(M_{\text {line }}\right)=\mathrm{CFE}_{\max } \times\left[1-\exp \left(1-2 M_{\text {line }} / M_{\text {line,crit }}\right)\right]$ with $\mathrm{CFE}_{\max }=15 \%$.

The global prestellar CMF expected in the framework of this toy model, and the CMFs expected in thermally transcritical filaments and slightly supercritical filaments are shown in Fig. 3 as a black solid, blue solid, and red solid curve, respectively. For comparison, the black, blue, and red histograms with error bars represent the corresponding CMFs observed with Herschel in Orion B (Könyves et al. 2019). A good, overall 
agreement can be seen. More importantly, it can be seen in Fig. 3 that the global prestellar CMF approaches the powerlaw shape of the FLMF at the high-mass end. We note that the empirical toy model described here is only meant to quantify the links between the FLMF and the CMF/IMF. It may also provide useful guidelines that will help develop a self-consistent physical model for the origin of the CMF/IMF in filaments in the future.

\section{Concluding remarks}

Our discussion of the Herschel observations in Sect. 2 indicates that both the filament line mass function (FLMF) and FMF are consistent with a steep Salpeter-like power law (dN/dlog $M_{\text {line }} \propto$ $M_{\text {line }}^{-1.6}$ and $\mathrm{d} N / \mathrm{d} \log M_{\text {tot }} \propto M_{\mathrm{tot}}^{-1.4}$, respectively) in the regime of thermally supercritical filaments $\left(M_{\text {line }}>16 M_{\odot} \mathrm{pc}^{-1}\right)$. This is a remarkable result since, in contrast, the mass distribution of molecular clouds and clumps is observed to be significantly shallower than the Salpeter power-law IMF, namely $\mathrm{d} N / \mathrm{d} \log M_{\mathrm{cl}} \propto M_{\mathrm{cl}}^{-0.7}$ (Blitz 1993; Kramer et al. 1998). Theoretically, the latter is reasonably well understood in terms of the mass function of both "bound objects on the largest self-gravitating scale" (Hopkins 2012) and non-self-gravitating structures (Hennebelle \& Chabrier 2008) generated by supersonic interstellar turbulence. Thus, filamentary structures in molecular clouds appear to differ from standard clumps in a fundamental way and may represent the key evolutionary step at which the steep slope of the prestellar CMF originates (and by extension that of the stellar IMF) (see Sect. 3).

In the context of the filament paradigm summarized in Sect. 1, we speculate that the observed FLMF arises from a combination of two effects. First, a spectrum of large-scale compression flows in the cold ISM produces a network of filamentary structures with an initial line mass distribution $\mathrm{d} N / \mathrm{d} \log M_{\text {line }} \propto M_{\text {line }}^{-1}$, determined by the power spectrum of interstellar turbulence (Iwasaki, priv. comm.). Turbulence is known to generate essentially self-similar, fractal structures in interstellar clouds (e.g., Larson 1992; Elmegreen \& Falgarone 1996), and this leads to a mass distribution of substructures with equal mass contribution per logarithmic interval of mass (i.e., $\mathrm{d} N / \mathrm{d} \log M \propto M^{-1}$ ) independent of the fractal dimension (Elmegreen 1997; Padoan \& Nordlund 2002). Second, thermally supercritical filaments accrete mass from the parent molecular cloud (Arzoumanian et al. 2013; Shimajiri et al. 2019b) due to their gravitational potential $\propto G M_{\text {line }}$ (Hennebelle \& André 2013). Therefore, they grow in mass per unit length at a rate $\dot{M}_{\text {line }} \propto \sqrt{G M_{\text {line }}}$ on a characteristic timescale $\tau_{\text {acc }}=M_{\text {line }} / \dot{M}_{\text {line }} \propto$ $\sqrt{M_{\text {line }}}$, while fragmenting and forming cores on a comparable timescale (see Heitsch 2013). The accretion timescale is on the order of 1-2 Myr for a Taurus-like filament with $M_{\text {line }} \sim$ $50 M_{\odot} \mathrm{pc}^{-1}$ (Palmeirim et al. 2013). As shown in Appendix C, starting from an initial line mass spectrum $\mathrm{d} N / \mathrm{d} \log M_{\text {line }} \propto M_{\text {line }}^{-1}$, this accretion process leads to a steepening of the distribution of supercritical masses per unit length on a similar timescale (Fig. C.2), and thus to a reasonable agreement with the observed FLMF (see Fig. C.3b).

Given the empirical toy model of Sect. 3 for the CMF produced by a collection of molecular filaments and its reasonably good match to observations (Fig. 3), we conclude that the filament paradigm for star formation provides a promising conceptual framework for understanding the origin of the prestellar $\mathrm{CMF}$ and by extension the stellar IMF.
Acknowledgements. We are grateful to S. Inutsuka and P. Hennebelle for many stimulating discussions. This work has received support from the European Research Council under the European Union's Seventh Framework Programme (ERC Advanced Grant Agreement no. 291294 - ORISTARS). We also acknowledge support from the French national programs of CNRS/INSU on stellar and ISM physics (PNPS and PCMI). DA and PP acknowledge support from FCT/MCTES through Portuguese national funds (PIDDAC) by grant UID/FIS/04434/2019. PP receives support from fellowship SFRH/BPD/110176/2015 funded by FCT (Portugal) and POPH/FSE (EC). YS is supported by NAOJ ALMA Scientific Research Grant Numbers 2017-04A. Our study has made use of data from the Herschel Gould Belt survey (HGBS) project, a Herschel Key Programme carried out by SPIRE Specialist Astronomy Group 3 (SAG 3), scientists of institutes in the PACS Consortium (CEA Saclay, INAFIFSI Rome and INAF-Arcetri, KU Leuven, MPIA Heidelberg), and scientists of the Herschel Science Centre (HSC).

\section{References}

Alves, J., Lombardi, M., \& Lada, C. J. 2007, A\&A, 462, L17

André, P., Men'shchikov, A., Bontemps, S., et al. 2010, A\&A, 518, L102

André, P., Di Francesco, J., Ward-Thompson, D., et al. 2014, in Protostars and

Planets VI, eds. H. Beuther, R. S. Klessen, C. P. Dullemond, et al., 27

Arzoumanian, D., André, P., Didelon, P., et al. 2011, A\&A, 529, L6

Arzoumanian, D., André, P., Peretto, N., \& Könyves, V. 2013, A\&A, 553, A119

Arzoumanian, D., André, P., Könyves, V., et al. 2019, A\&A, 621, A42

Bate, M. R., Bonnell, I. A., \& Bromm, V. 2003, MNRAS, 339, 577

Blitz, L. 1993, in Protostars and Planets III, eds. E. H. Levy, \& J. I. Lunine, 125 Bonnell, I. A., Bate, M. R., Clarke, C. J., \& Pringle, J. E. 2001, MNRAS, 323, 785

Bonnor, W. B. 1956, MNRAS, 116, 351

Chabrier, G. 2005, in The Initial Mass Function 50 Years Later, eds. E. Corbelli,

F. Palla, \& H. Zinnecker, Astrophys. Space Sci. Lib., 327, 41

Elmegreen, B. G. 1997, ApJ, 486, 944

Elmegreen, B. G., \& Falgarone, E. 1996, ApJ, 471, 816

Fiege, J. D., \& Pudritz, R. E. 2000, MNRAS, 311, 85

Fischera, J., \& Martin, P. G. 2012, A\&A, 542, A77

Heitsch, F. 2013, ApJ, 769, 115

Hennebelle, P., \& André, P. 2013, A\&A, 560, A68

Hennebelle, P., \& Chabrier, G. 2008, ApJ, 684, 395

Hopkins, P. F. 2012, MNRAS, 423, 2016

Inutsuka, S.-I. 2001, ApJ, 559, L149

Inutsuka, S.-I., \& Miyama, S. M. 1997, ApJ, 480, 681

Inutsuka, S.-I., Inoue, T., Iwasaki, K., \& Hosokawa, T. 2015, A\&A, 580, A49

Koch, E. W., \& Rosolowsky, E. W. 2015, MNRAS, 452, 3435

Könyves, V., André, P., Men'shchikov, A., et al. 2015, A\&A, 584, A91

Könyves, V., André, P., Arzoumanian, D., et al. 2019, A\&A, submitted

Kramer, C., Stutzki, J., Rohrig, R., \& Corneliussen, U. 1998, A\&A, 329, 249

Kroupa, P. 2001, MNRAS, 322, 231

Ladjelate, B., André, P., Könyves, V., et al. 2019, A\&A, submitted

Larson, R. B. 1985, MNRAS, 214, 379

Larson, R. B. 1992, MNRAS, 256, 641

Larson, R. B. 2005, MNRAS, 359, 211

Lee, Y.-N., Hennebelle, P., \& Chabrier, G. 2017, ApJ, 847, 114

Mac Low, M.-M., \& Klessen, R. S. 2004, Rev. Mod. Phys., 76, 125

Marsh, K. A., Kirk, J. M., André, P., et al. 2016, MNRAS, 459, 342

McKee, C. F., \& Tan, J. C. 2003, ApJ, 585, 850

Men'shchikov, A. 2013, A\&A, 560, A63

Molinari, S., Swinyard, B., Bally, J., et al. 2010, A\&A, 518, L100

Motte, F., André, P., \& Neri, R. 1998, A\&A, 336, 150

Offner, S. S. R., Clark, P. C., Hennebelle, P., et al. 2014, in Protostars and Planets

VI, eds. H. Beuther, R. S. Klessen, C. P. Dullemond, et al., 53

Padoan, P., \& Nordlund, Å. 2002, ApJ, 576, 870

Palmeirim, P., André, P., Kirk, J., et al. 2013, A\&A, 550, A38

Press, W. H., Teukolsky, S. A., Vetterling, W. T., \& Flannery, B. P. 1992,

Numerical Recipes in FORTRAN. The Art of Scientific Computing, 2nd edn.

(Cambridge: Cambridge University Press)

Roy, A., André, P., Arzoumanian, D., et al. 2015, A\&A, 584, A111

Salpeter, E. E. 1955, ApJ, 121, 161

Schisano, E., Rygl, K. L. J., Molinari, S., et al. 2014, ApJ, 791, 27

Shimajiri, Y., André, P., Ntormousi, E., et al. 2019a, A\&A, submitted

Shimajiri, Y., André, P., Palmeirim, P., et al. 2019b, A\&A, 623, A16

Sousbie, T. 2011, MNRAS, 414, 350

Tomisaka, K. 2014, ApJ, 785, 24

Zinnecker, H. 1982, Annals of the New York Academy of Sciences, 395, 226 


\section{Appendix A: Distribution of filament lengths}
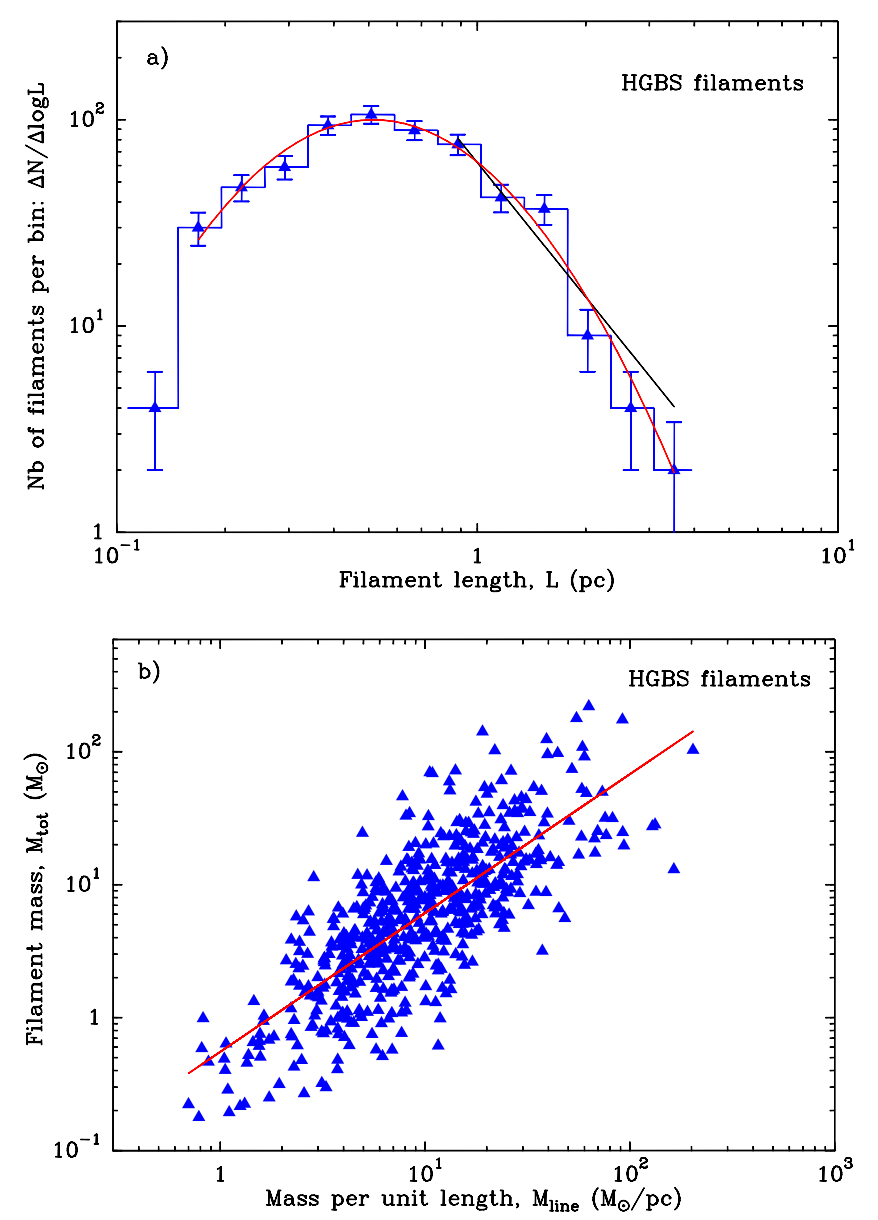

Fig. A.1. Panel $a$ : differential distribution of lengths for the sample of 599 robust HGBS filaments identified by Arzoumanian et al. (2019). The red curve shows a lognormal fit to the overall distribution, the black solid line segment a power-law fit for filaments longer than $0.9 \mathrm{pc}$. Panel $b$ : total mass $M_{\mathrm{tot}}$ against average mass per unit length $M_{\text {line }}$ for the same sample of filaments as in the top panel. A strong linear correlation is observed between $\log M_{\text {tot }}$ and $\log M_{\text {line }}$ (with a Pearson correlation coefficient of $\sim 77 \%$ ). The red line shows the best-fit linear relation, $M_{\mathrm{tot}}=M_{\text {line }} \times L_{\mathrm{eff}}$, consistent with a typical effective length $L_{\mathrm{eff}} \sim 0.55 \mathrm{pc}$ in the filament sample of Arzoumanian et al. (2019).

In this appendix, we show the distribution of filament lengths in the Arzoumanian et al. (2019) sample (Fig. A.1a) and the linear correlation between filament mass and filament mass per unit length (Fig. A.1b), consistent with a roughly uniform length $L_{\text {eff }} \sim 0.55$ pc independent of $M_{\text {line }}$.

\section{Appendix B: Observational constraints on the core mass function in individual filaments}

The form of the prestellar CMF produced by a single filament of line mass $M_{\text {line }}$, denoted $f_{M_{\text {line }}}(m)$ in the text, is the most uncertain element of the empirical toy model described in Sect. 3 for the CMF/IMF. For statistical reasons, observational estimates of CMFs in individual filaments are difficult owing to the relatively low number of cores per filament (but see the promising ALMA results of Shimajiri et al. 2019a for the massive filament in NGC 6334). Observations nevertheless indicate that the median prestellar core mass increases roughly linearly with the line mass of the parent filament and that the dispersion
Equivalent line mass of parent filament $\left(\mathrm{M}_{\odot} / \mathrm{pc}\right)$

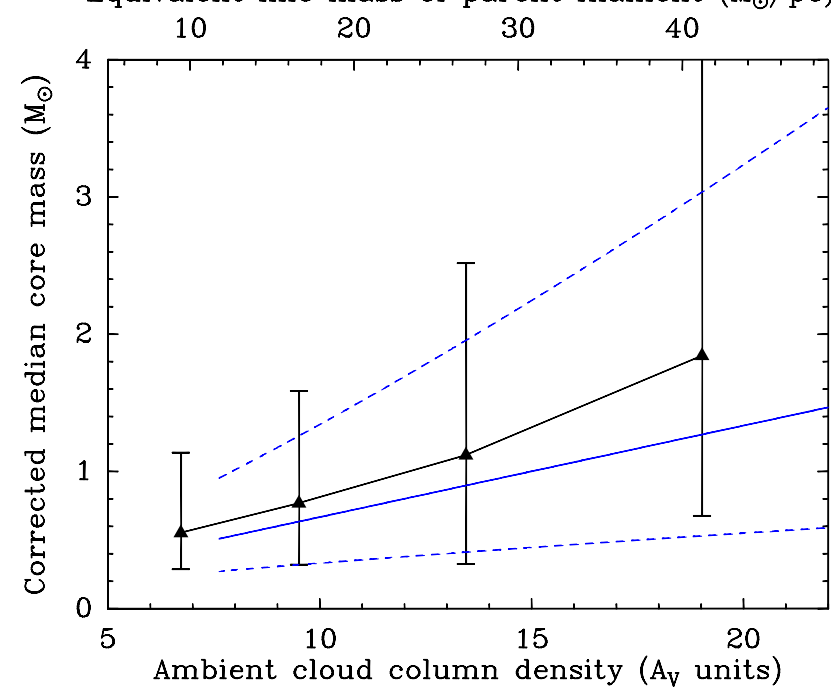

Fig. B.1. Median prestellar core mass vs. background column density as observed in the Orion B region after correction for incompleteness effects (black triangles, from Könyves et al. 2019), compared to the prediction of the toy model described in Sect. 3 (blue solid line). The error bars correspond to the inter-quartile range in observed masses for each bin of background column density. The two dashed blue lines mark the inter-quartile range expected in the context of the toy model (see Eq. (2)).

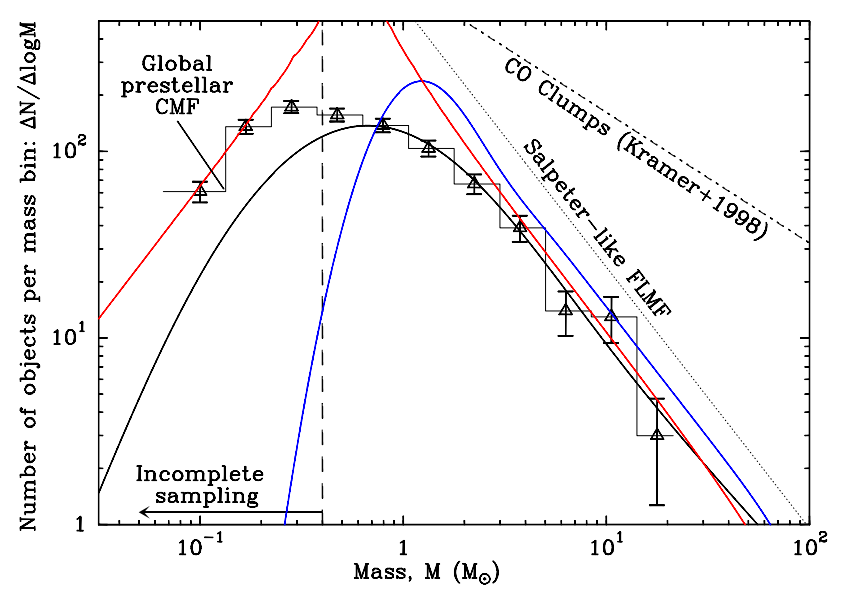

Fig. B.2. Comparison of the prestellar CMF observed in Orion B (black triangular data points and histogram from Könyves et al. 2019; see also Fig. 3) with the global prestellar CMFs expected in the toy model of Sect. 3, under three assumptions about the shape of the CMF generated by a single filament of line mass $M_{\text {line }}$ : (i) lognormal $f_{M_{\text {line }}}(m)$ distribution (Eq. (2)) with $\sigma_{M_{\text {line }}}=\sqrt{0.4^{2}+0.3\left[\log \left(M_{\mathrm{BE}, \text { eff }} / M_{\mathrm{BE}, \mathrm{th}}\right)\right]^{2}}$ (fiducial case, black curve); (ii) lognormal $f_{M_{\text {line }}}(m)$ distribution (Eq. (2)) with fixed $\sigma_{M_{\text {line }}}=0.2$ independent of $M_{\text {line }}$ (blue curve); and (iii) broken power law, $f_{M_{\text {line }}}(m) \propto m^{6}$ for $m<M_{\mathrm{BE}, \text { th }}, f_{M_{\text {line }}}(m)=$ constant for $M_{\mathrm{BE}, \text { th }} \leq m<M_{\mathrm{BE}, \text { eff }}, f_{M_{\text {line }}}(m) \propto m^{-4}$ for $m>M_{\mathrm{BE} \text {,eff }}$ (red curve). For reference, the black dotted line displays the Salpeter-like power-law FLMF, $\mathrm{d} N / \mathrm{d} \log M_{\text {line }} \propto M_{\text {line }}^{-1.4}$, observed in the supercritical line mass regime (see Fig. 1), and the black dash-dotted line shows the typical mass distribution of CO clumps (Kramer et al. 1998).

in core masses also increases with $M_{\text {line }}$ ((Könyves et al. 2019), see also Fig. B.1). In agreement with this observational trend, the qualitative arguments presented in Sect. 3 suggest that the characteristic prestellar core mass should scale with the effective Bonnor-Ebert mass $M_{\mathrm{BE}, \text { eff }}$ in the parent filament and that the 
dispersion in core masses may scale with the ratio $M_{\mathrm{BE}, \mathrm{eff}} / M_{\mathrm{BE}, \mathrm{th}}$. The blue lines in Fig. B.1 show how the median core mass and the dispersion in core masses vary with $M_{\text {line }}$ in the toy model of Sect. 3, which assumes a lognormal shape for $f_{M_{\text {line }}}(m)$ with standard deviation $\sigma_{M_{\text {line }}}=\sqrt{0.4^{2}+0.3\left[\log \left(M_{\mathrm{BE}, \text { eff }} / M_{\mathrm{BE}, \mathrm{th}}\right)\right]^{2}}$. The latter expression for $\sigma_{M_{\text {line }}}$ corresponds to the quadratic sum of two terms: the first term represents the intrinsic spread in the core masses generated by transcritical filaments (which have $\left.M_{\mathrm{BE}, \text { eff }} / M_{\mathrm{BE}, \mathrm{th}} \sim 1\right)$, while the second term represents the spread in characteristic fragmentation masses within supercritical but virialized filaments (which have $M_{\mathrm{BE} \text {,eff }} / M_{\mathrm{BE} \text {,th }}>1$; see Sect. 3). It can be seen in Fig. B.1 that these simple assumptions about $f_{M_{\text {line }}}(m)$ and $\sigma_{M_{\text {line }}}$ match the observational constraints reasonably well.

We also note that the high-mass end of the global prestellar CMF in our toy model is primarily driven by the power-law shape of the FLMF and depends only weakly on the detailed form assumed for $f_{M_{\text {line }}}(m) \equiv \mathrm{d} N_{M_{\text {line }}} / \mathrm{d} \log m$. This is illustrated in Fig. B.2 which shows the model global CMFs for three different assumptions about $f_{M_{\text {line }}}(m)$, compared to the prestellar CMF observed in Orion B (Könyves et al. 2019, see also Fig. 3). It can be seen that the three model CMFs are consistent with a Salpeterlike power law at the high-mass end and only differ significantly at the low-mass end.

\section{Appendix C: Toy accretion model for the filament line mass function}

As mentioned in Sect. 4, thermally supercritical filaments are believed to accrete mass from their parent molecular cloud (Arzoumanian et al. 2013; Shimajiri et al. 2019b) owing to their gravitational potential $\propto G M_{\text {line }}$ (Heitsch 2013; Hennebelle \& André 2013). This leads to an accretion rate $\dot{M}_{\text {line }} \propto \sqrt{G M_{\text {line }}}($ see Palmeirim et al. 2013), and therefore to a simple differential equation of the form

$\frac{\mathrm{d} M_{\text {line }}}{\mathrm{d} t}=A M_{\text {line }}^{1 / 2}$,

where $A$ is a positive constant. This equation can be easily integrated to give the time evolution of the line mass due to gravitational accretion:

$M_{\text {line }}(t)=\left[M_{\text {line }}(0)^{1 / 2}+\frac{A}{2} t\right]^{2}$.

If we choose to express time $\tilde{t}$ in units of the time needed to increase the line mass of an initially critical filament by a factor of 4, Eq. (C.2) can be written in the following form:

$M_{\text {line }}(\tilde{t})=\left[M_{\text {line }}(0)^{1 / 2}+M_{\text {line,crit }}^{1 / 2} \tilde{t}\right]^{2}$.

In these units, the characteristic instantaneous accretion timescale is

$\tilde{\tau}_{\text {acc }}=M_{\text {line }} / \dot{M}_{\text {line }}=\frac{1}{2}\left(\frac{M_{\text {line }}}{M_{\text {line }, \text { crit }}}\right)^{1 / 2} \propto \sqrt{M_{\text {line }}}$.

In absolute terms, the characteristic accretion timescale is on the order of 1-2 Myr for a Taurus-like filament with $M_{\text {line }} \sim$ $50 M_{\odot} \mathrm{pc}^{-1} \sim 3 M_{\text {line,crit }}$ (Palmeirim et al. 2013; Shimajiri et al. $2019 b)$. Figure C. 1 shows the time evolution $M_{\text {line }}(\tilde{t})$ predicted by this simple accretion model for five values of the initial line mass $M_{\text {line }}(0)$.

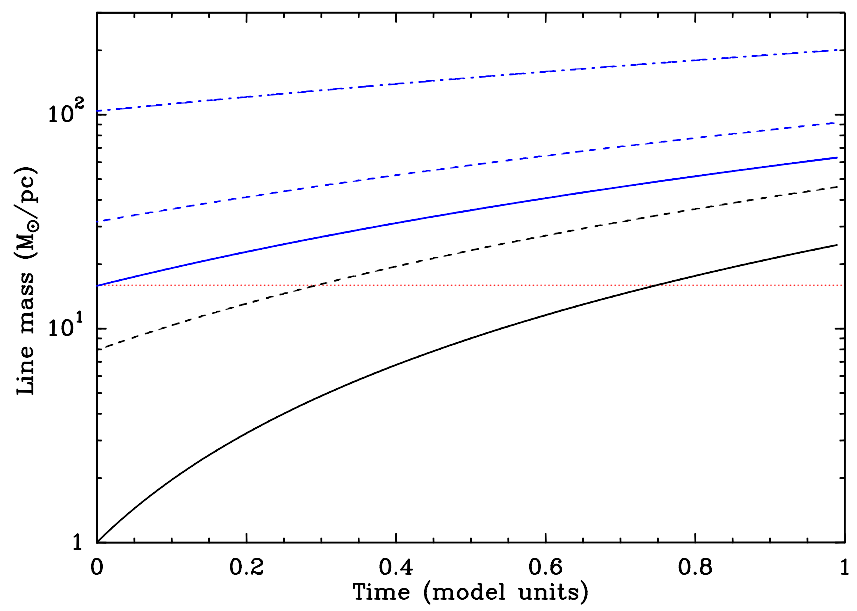

Fig. C.1. Evolution of the mass per unit length of filaments according to the toy gravitational-accretion model described in the text, for five values of the initial line mass at $t=0: M_{\text {line }}(0)=1 M_{\odot} \mathrm{pc}^{-1}$ (black solid curve), $M_{\text {line }}(0) \sim 8 M_{\odot} \mathrm{pc}^{-1}$ (half critical, black dashed curve), $M_{\text {line }}(0) \sim 16 M_{\odot} \mathrm{pc}^{-1}$ (critical, blue solid curve), $M_{\text {line }}(0) \sim 32 M_{\odot} \mathrm{pc}^{-1}$ (twice critical, blue dashed curve), $M_{\text {line }}(0) \sim 100 M_{\odot} \mathrm{pc}^{-1}$ (highly supercritical, blue dash-dotted curve). The red dotted horizontal line marks the critical line mass of $\sim 16 M_{\odot} \mathrm{pc}^{-1}$. Time is normalized in such a way that a critical filament with $M_{\text {line }}=M_{\text {line,crit }}$ at $\tilde{t}=0$ has $M_{\text {line }}=4 \times M_{\text {line,crit }}$ at $\tilde{t}=1$ (solid blue curve), corresponding to $\sim 1-2 \mathrm{Myr}$.

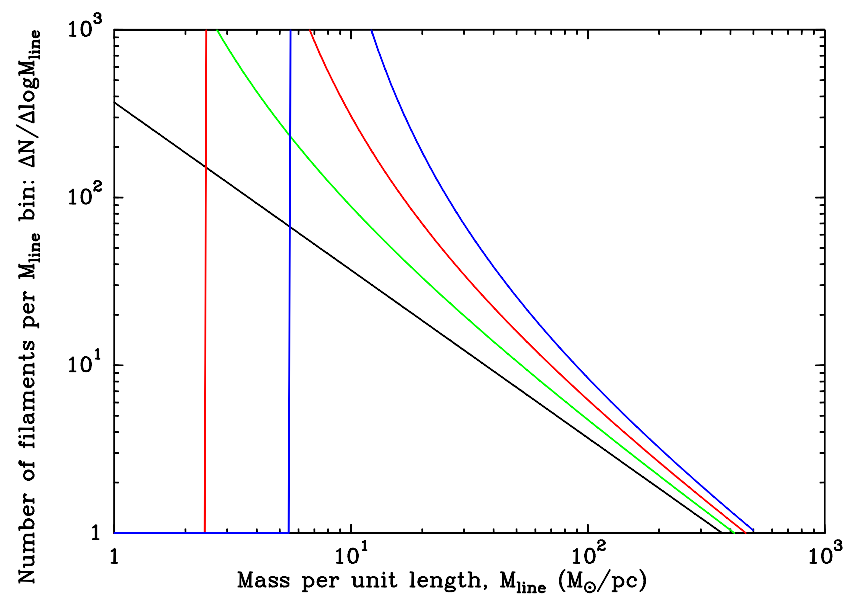

Fig. C.2. Evolution of the FLMF in the context of the proposed toy accretion model. The black solid line shows the initial power-law FLMF ( $\left.\mathrm{d} N / \mathrm{d} \log M_{\text {line }} \propto M_{\text {line }}^{-1}\right)$ determined by interstellar turbulence. The green, red, and blue solid curves show the model FLMF at three time steps, $\tilde{t}=0.2, \tilde{t}=0.4, \tilde{t}=0.6$ after the accretion process is "switched on" at $\tilde{t}=0$, where $\Delta \tilde{t}=0.4$ roughly corresponds to the time it takes for a critical filament to double its mass per unit length ( 0.5-1 Myr). The median logarithmic slope of the model FLMF for $16<M_{\text {line }}<500 M_{\odot} \mathrm{pc}^{-1}$ is $-1,-1.14,-1.30$, and -1.50 at $\tilde{t}=0$, $\tilde{t}=0.2, \tilde{t}=0.4$, and $\tilde{t}=0.6$, respectively. The vertical red and blue lines correspond to the line mass $M_{\text {line,crit }} \tilde{t}^{2}$ accreted by filaments with $M_{\text {line }}(0) \approx 0$ at $\tilde{t}=0.4$ and $\tilde{t}=0.6$, respectively.

In the context of this model, we can derive the time evolution of the FLMF following an approach similar to that employed by Zinnecker (1982) in his toy model of the IMF based on Bondi-Hoyle accretion (for which $\dot{M}_{\star} \propto M_{\star}^{2}$ ). Mass conservation implies that the cumulative distribution of line masses at time $\tilde{t}, N_{\tilde{t}}\left[>M_{\text {line }}\right]$ is related to the initial distribution of line 

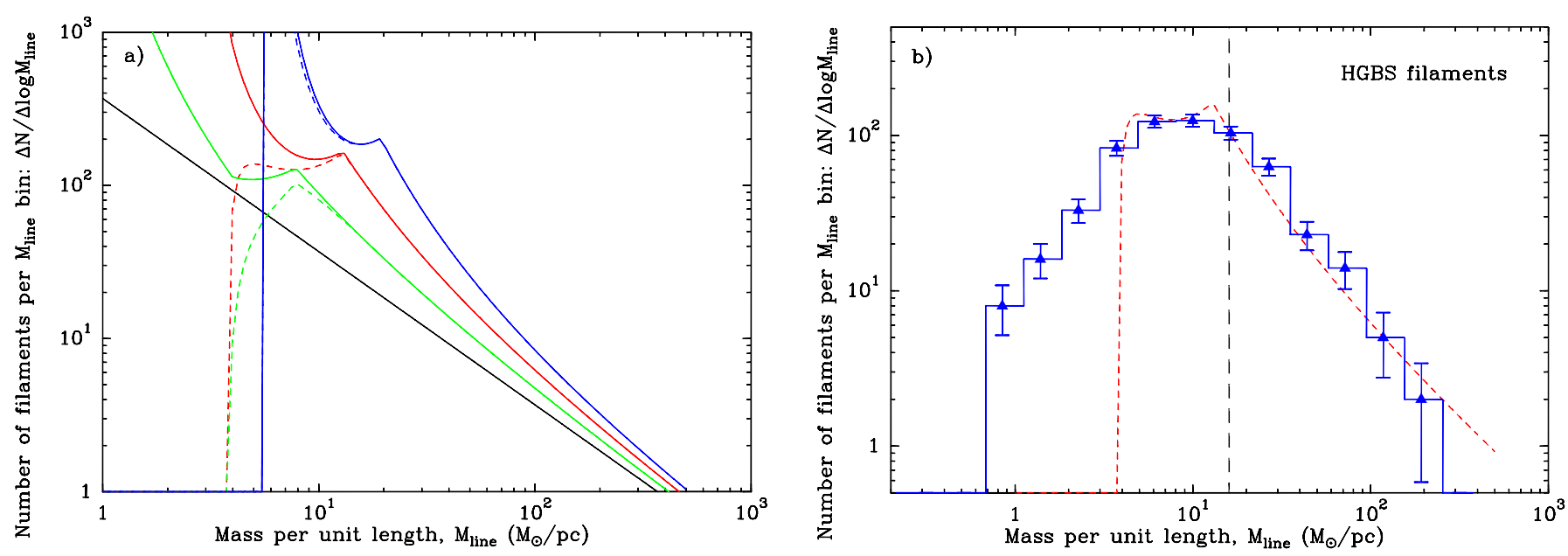

Fig. C.3. Panel a: evolution of the FLMF according to the improved version of our model where filaments accrete in the same way as in Fig. C.2, but subcritical filaments with $M_{\text {line }}<4 M_{\odot} \mathrm{pc}^{-1}$ decay on a timescale $\tilde{\tau}_{\text {decay }}=0.15$ at the same time. The black solid line shows the initial powerlaw FLMF $\left(\mathrm{d} N / \mathrm{d} \log M_{\text {line }} \propto M_{\text {line }}^{-1}\right)$ determined by interstellar turbulence. The green, red, and blue solid curves show the model FLMF at three time steps, $\tilde{t}=0.2, \tilde{t}=0.4, \tilde{t}=0.6$ after the accretion process is "switched on" at $\tilde{t}=0$, where $\tilde{t}=0.4$ roughly corresponds to $\sim 0.5-1 \mathrm{Myr}$. The vertical red and blue lines are the same as in Fig. C.2. The green, red, and blue dashed curves show the same model FLMF taking estimated incompleteness effects into account in the subcritical line mass regime. Panel $b$ : comparison of the model FLMF including incompleteness effects at $\tilde{t}=0.4$ (dashed red curve) with the observed FLMF from Fig. 1a (blue histogram).

masses by

$N_{\tilde{t}}\left[>M_{\text {line }}\right]=N_{0}\left[>M_{0, \tilde{t}}\left(M_{\text {line }}\right)\right] \equiv \int_{M_{0, \tilde{t}}\left(M_{\text {line }}\right)}^{+\infty} \frac{\mathrm{d} N_{0}}{\mathrm{~d} M_{\text {line }}^{0}} \mathrm{~d} M_{\text {line }}^{0}$,

where $M_{0, \tilde{t}}\left(M_{\text {line }}\right)=\left(M_{\text {line }}^{1 / 2}-M_{\text {line,crit }}^{1 / 2} \tilde{t}\right)^{2}$ represents the initial line mass of a filament with line mass $M_{\text {line }}$ at time $\tilde{t}$. The differential FLMF at time $\tilde{t}$ can then be obtained by taking the derivative of Eq. (C.5) with respect to $M_{\text {line }}$,

$\frac{\mathrm{d} N_{\tilde{t}}}{\mathrm{~d} M_{\text {line }}} \equiv-\frac{\mathrm{d} N_{\tilde{t}}\left[>M_{\text {line }}\right]}{\mathrm{d} M_{\text {line }}}=\frac{\mathrm{d} N_{0}}{\mathrm{~d} M_{\text {line }}^{0}}\left[M_{0, \tilde{t}}\left(M_{\text {line }}\right)\right] \times \frac{\mathrm{d} M_{0, \tilde{t}}}{\mathrm{~d} M_{\text {line }}}$,

which leads to

$\frac{\mathrm{d} N_{\tilde{t}}}{\mathrm{~d} M_{\text {line }}}\left(M_{\text {line }}\right)=\frac{\mathrm{d} N_{0}}{\mathrm{~d} M_{\text {line }}^{0}}\left[M_{0, \tilde{t}}\left(M_{\text {line }}\right)\right] \times\left(1-\frac{M_{\text {line,crit }}^{1 / 2}}{M_{\text {line }}^{1 / 2}} \tilde{t}\right)$.

The latter can also be written as

$\frac{\mathrm{d} N_{\tilde{t}}}{\mathrm{~d} M_{\text {line }}}\left(M_{\text {line }}\right)=\frac{\mathrm{d} N_{0}}{\mathrm{~d} M_{\text {line }}^{0}}\left[M_{0, \tilde{t}}\left(M_{\text {line }}\right)\right] \times\left(\frac{M_{\text {line }}}{M_{0, \tilde{t}}}\right)^{1 / 2}$.

Starting from an initial power-law FLMF d $N / \mathrm{d} \log M_{\text {line }} \propto M_{\text {line }}^{-1}$ determined by interstellar turbulence (Sect. 4), the resulting
FLMF is shown at three time steps, $\tilde{t}=0.2, \tilde{t}=0.4, \tilde{t}=0.6$, and compared to the initial power law at $\tilde{t}=0$ in Fig. C.2. It can be seen that the accretion process steepens the model FLMF with time, making it more consistent with the observed FLMF of Fig. 1a in the supercritical regime than the initial power-law FLMF. In particular, the median logarithmic slope of the model FLMF in the range of line masses $16<M_{\text {line }}<500 M_{\odot} \mathrm{pc}^{-1}$ is between -1.5 and -1.3 (i.e., Salpeter-like) at $\tilde{t} \sim 0.2-0.4$, which is less than $1 \mathrm{Myr}$ after the onset of accretion, in good agreement with the observed FLMF which has a logarithmic slope of $-1.4 \pm 0.1$ (Fig. 1a).

The model FLMF nevertheless quickly diverges near $M_{\text {line }}=$ $M_{\text {line,crit }} \tilde{t}^{2}$, due to an accumulation of filaments with very low initial masses per unit length (i.e., $M_{\text {line }}(0) \approx 0$ ) whose $M_{\text {line }}(\tilde{t})$ is entirely built up by gravitational accretion. This is not very physical since filaments that are highly subcritical initially $\left(M_{\text {line }}(0) \ll M_{\text {line,crit }} / 2\right)$ are not self-gravitating and are unlikely to gravitationally accrete mass from the ambient cloud. Instead, these filaments may disperse on a sound crossing time unless they are pressure-confined. In Fig. C.3, we present an improved version of the same accretion model where the number of subcritical filaments with $M_{\text {line }}<4 M_{\odot} \mathrm{pc}^{-1}$ decay on a characteristic timescale $\tilde{\tau}_{\text {decay }}=0.15$, at the same time as the filaments accrete mass on the timescale $\tilde{\tau}_{\text {acc }}$ given by Eq. (C.4). It can be seen that this modified model provides a better match to the observed FLMF (Fig. C.3b), especially when incompleteness effects are taken into account in the subcritical line mass regime (dashed curves in Fig. C.3a). 